\title{
El qué, el quién, el por qué, el dónde, el cuándo y el cómo de la prevención primaria
}

\section{Thomas P. Gullotta}
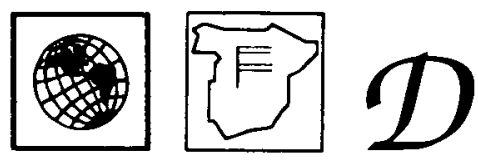

En el artículo se propone una definición de "prevención primaria» a partir de la cual se analizará: la población a la que van dirigidas las actividades de este tipo de prevención, la necesidad de la prevención primaria no sólo desde el punto de vista de la salud sino también desde el punto de vista económico, los espacios y momentos en que se lleva a cabo la prevención primaria, y los instrumentos de intervención que le son propios.

Durante la primavera de 1993, tuve la oportunidad de participar en un grupo asesor para el Center for Substance Abuse Prevention. Procedentes de varias organizaciones profesionales, los miembros de este grupo dedicaron tres difíciles días a intentar asesorar a un grupo de contratistas sobre el desarrollo de currículos de prevención para disciplinas como la asistencia social, la enfermetía y la odontología, así como sobre el personal de centros de salud comunitarios y especialistas en rehabilitación. Utilizo deliberadamente la palabra «difícil» porque pocos de los miembros de este grupo asesor pudieron ponerse de acuerdo ni siquiera en llegar a una definición común de la prevención primaria. Al contrario, obstaculizados por las limitaciones de nuestros paradigmas educativos y nuestras experiencias prácticas, cada uno de nosotros definía la prevención para que abarcara, de una manera general, sus actividades profesionales del momento.

Varios meses después tuve una experiencia similar, aunque más breve, en una conferencia del National Institute of Health. Esta conferencia, anunciada como «Investigación de la prevención de enfermedades en el NIH: Ura agenda común», me dio la sensación de que la tan cacareada «década del cerebro» del NIH se había reconvertido al lenguaje más actual y políticamente correcto de la prevención. Estas experiencias recientes sirven de motivación para emprender la redacción de este artículo. Se trata de un intento de describir la prevención primaria independientemente de cualquier disciplina o campo. Se trata de un intento de discutir la prevención primaria de una manera que ilustre sus principios básicos. 


\section{¿QUE ES LA PREVENCION PRIMARIA?}

La prevención primaria puede definirse como una serie de esfuerzos planificados para reducir (prevenir) la incidencia de casos nuevos de comportamiento disfuncional en una población que aún no muestre señales de este comportamiento y para fomentar (promover) conductas que contribuyan al mantenimiento de comportamientos funcionales.

Esta definición evita el empleo de palabras como enfermedad y salud, aunque en párrafos posteriores utilizaré estas y orras palabras intercambiándolas con el comportamiento funcional y disfuncional. Palabras como enfermedad o salud con frecuencia pueden urilizarse para implicar un origen biológico del comportamiento. Aunque no negaré que algunos comportamientos disfuncionales pueden contener un componente biológico, no ocurre así con la mayoría de ellos. E incluso los comportamientos que han atraído el mayor interés biológico (como el alcoholismo y la depresión) siguen siendo blancos escurridizos para los geneticistas que, incluso en el mejor de sus imperfectos estudios, deben continuar atribuyendo la mitad o más de la varianza a variables ambientales (véanse Holden, 1987, 1991; Peele, 1986; Plomin, 1990). En cambio, los comportamientos disfuncionales suelen ser casi siempre el resultado de un deterioro de las relaciones interpersonales o de las relaciones entre una persona y la sociedad (Albee, 1983, 1987).

\section{¿A QUIEN SE DIRIGEN LAS ACTIVIDADES DE LA PREVENCION PRI- MARIA?}

\section{La prevención primaria se centra en poblaciones saludables}

El por qué del quién puede encontrarse en el qué de la prevención primaria. Es decir, la prevención primaria se ocupa de reducir la incidencia de nuevos casos de comportamiento disfuncional en una sociedad, fomentando al mismo tiempo estilos de vida que induzcan en esa sociedad comportamientos funcionales. El tratamiento no es prevención. La rehabilitación no es prevención.

¿Significa esto que individuos con lesiones cerebrales recientes no pueden beneficiarse de iniciativas de prevención primaria? En modo alguno. Si la iniciativa consiste en promover la comprensión y la aceptación de individuos con discapacidades en la sociedad más amplia, entonces se trata de una población más que adecuada con la que trabajar. Si la iniciativa es un conjunto de actividades centradas en un grupo (que impliquen educación, promover la competencia, la autoayuda y el cambio comunitario) para prevenir la depresión clínica que con frecuencia sigue a lesiones graves de esta naturaleza, entonces esta población es, nuevamente, una población más que apropiada con la que trabajar. Por cierto, obsérvese en el primer ejemplo que el objetivo del comportamiento modificado es el público en general.

Otro aspecto relacionado con el quién se encuentra en la palabra «sociedad». Para la salud mental, la salud pública y otras disciplinas de asistencia profesional, la prevención se centra en el grupo, no en el individuo (Klein y Goldston, 1977). Estos esfuerzos proactivos dirigidos hacia la población y emprendidos por disciplinas de asistencia profesional, se destinan a estimular a otras personas a actuar sobre una base individual para promover comportamientos funcionales. La prevención se produce equipando a enseñantes, sacerdotes, líderes juveniles, camareros, peluqueros, periodistas, estudiantes, padres, amigos y otras personas con la capacidad, las habilidades y el conocimiento para promover comportamientos funcionales en sí mismos y en otras personas. Desde la perspectiva interaccionista simbólica, cuando los profesionales de la salud mental intercambian sus papeles asistenciales por otros papeles como los de padre, cónyuge, amigo o líder juvenil, tienen la oportunidad (al actuar 
como ciudadanos particulares) de promover comportamientos funcionales para sí mismos y para otros.

Por cierto, volviendo al ejemplo anterior de una persona con una lesión cerebral reciente, todos los grupos formados en la prevención mencionados anteriormente hubieran fomentado el comportamiento de que llevar puesto un casco al ir en bicicleta, a caballo, en moto o sobre un monopatín era una acción sensata y prudente. Evitar una lesión cerebral incapacitadora es preferible a prevenir la depresión que con frecuencia sigue a estas lesiones o a promover la aceptación social de individuos que han experimentado un suceso tan desafortunado.

\section{¿POR QUE ES NECESARIA LA PREVENCION PRIMARIA?}

\section{La prevención primaria es necesaria tanto en el plano de la salud como en el plano económico}

Desde el viejo refrán de que más vale prevenir que curar a la realidad de que los ciudadanos estadounidenses ya no pueden soportar el coste de una industria de la salud pública en perpetua expansión e hipnotizada por las tecnologías punteras, la prevención tiene mucha razón de ser. Como se observaba anteriormente, la mayoría de los comportamientos disfuncionales (enfermedades) que afectan a los individuos son decisiones relacionadas con el estilo de vida. Se trata de decisiones que pueden y deben orientarse hacia comportamientos más funcionales (más sanos). Como decía la National Commission on Children (1991, pp. 126-127) en su informe Beyond Rhetoric. A New American Agenda for Children and Families:

«La desnutrición, la obesidad y la incidencia de muchas enfermedades están relacionadas con la alimentación. Enfermedades de tranmisión sexual, accidentes y lesiones, y deficiencias mentales y físicas, son directamente atribuibles a una actividad sexual precoz sin protección, al alcohol y otras drogas y a conductas delictivas ... En realidad, un mejor control de un número limitado de factores de riesgo ... puede prevenir por lo menos un 40 por ciento de todas las muertes prematuras, una tercera parte de todos los casos de incapacidad a corto plazo y dos terceras partes de todos los casos de incapacidad crónica. Los cambios en los comportamientos relacionados con la salud también pueden reducir costes médicos y limitar pérdidas de productividad. Las enfermedades atribuibles al tabaco cuestan a los individuos y a la sociedad más de 65 mil millones de dólares al año. El coste total del abuso de alcohol y otras drogas sobrepasa los 110 mil millones de dólares al año.»

(National Commission on Children, 1991: pp. 126-127)

Invertir en la prevención no significa abandonar el tratamiento de la salud mental o los servicios de rehabilitación. Ciertamente, como han destacado George Albee (1983, 1985, y Gullotta, 1986) y otros (Commitree on Preventive Psychiatry, 1980), los estudios epidemiológicos sugieren que, en cualquier año dado, el 15 por ciento de la población de los Estados Unidos se encuentra emocionalmente enferma de gravedad (empleando el lenguaje de la industria de la salud pública). Con una población para los Estados Unidos estimada en 255 millones de personas, esto significa que más de 38 millones de personas necesitan ayuda cada año. Sin embargo, la capacidad de rehabilitación y tratamiento de los Estados Unidos no da más que para una minúscula fracción de esta cantidad. Si la prevención únicamente redujera esta población de individuos afectados en un 20 por ciento de casos al año, es decir, en 7.650.000 personas, sobrepasaría la capacidad total de tratamiento de los Estados Unidos para cualquier año dado. Dado que no todas las intervenciones clínicas tienen éxito ni se dirigen a pacientes considerados como los más gravemente enfermos, la relación entre costes y beneficios de la prevención se manifiesta con mucha claridad. Pero aún más importante que el hecho de que la relación entre costes y beneficios favorece a la prevención es la realidad de que millones de niños y adultos pueden evitar un sufrimiento innecesario. 


\section{¿DONDE SE PRODUCE LA PREVENCION PRIMARIA?}

\section{Las oportunidades para la prevención se encuentran en todas partes}

La prevención se produce en el hogar cuando un padre que utiliza las habilidades aprendidas en una clase de eficacia paterna aplica estos conocimientos para corregir el comportamiento de un niño sin recurrir a medidas violentas. La prevención se produce en un grupo de individuos discapacitados cuando forman una comunidad de autoayuda en la que sus miembros se sienten integrados, son apreciados y hacen contribuciones significativas a la existencia del grupo. La prevención se da con la Americans with Disabilities Act, con la legislación sobre derechos civiles y con la legislación sobre el abandono familiar. Se acentúa con las leyes sobre la obligatoriedad del cinturón de seguridad y del casco, y sobre el control de la posesión de armas. La prevención se produce cuando un individuo atiende seriamente a las advertencias colocadas en los paquetes de cigarrillos o en las botellas de alcohol. También se produce cuando a un niño se le hace ver la necesidad de ir al hospital. Lo mismo ocurre cuando a los padres de este niño se les permite acompañarle durante su estancia en el hospital. La prevención se da cuando los entrenadores deportivos emplean las aptitudes que han aprendido (Danish, 1990) para fomentar la competencia social entre sus alumnos o cuando las escuelas y los padres facilitan la entrada de estudiantes procedentes de traslado en nuevos entornos educativos (Jason, Kurasaki, Neuson y García, 1993).

Las oportunidades que tiene el ciudadano para realizar intervenciones preventivas se encuentran por doquier. Sin embargo, para los profesionales de la salud pública, la prevención es intervenir de una manera planificada, con la tecnología de prevención adecuada, para efectuar cambios colectivos en una población hasta el momento no afectada. Esto es el dónde de la prevención.

\section{¿CUANDO SE PRODUCE LA PREVENCION?}

\section{La prevención se produce antes de la aparición del comportamiento disfuncional y continúa durante toda la vida}

Las intervenciones universales como las campañas contra la polio y el sarampión, y otras vacunas administradas durante la primera infancia, son ejemplos de acciones preventivas realizadas antes de la aparición de enfermedades infantiles graves. El uso de cinturones de seguridad y air bags para reducir la incidencia de lesiones en los accidentes de tráfico también sirve como ejemplo. La atención prenatal médica y nutricional, y los cursillos de enseñanza, también constituyen ejemplos de actividad preventiva orientada a garantizar resultados saludables para el niño y la madre.

En base a todo lo dicho, no solo es aceptable, sino también deseable, llevar a cabo intervenciones preventivas con grupos vulnerables o de riesgo elevado. Por ejemplo, antes he empleado la expresión «intervención universal» para referirme a las vacunas de la primera infancia. Sin embargo, para los niños pobres, inmigrantes o sin hogar, la probabilidad de que reciban estas vacunas no es total en modo alguno. En un sistema de salud pública diseñado para las clases medias y superiores, el acceso a la asistencia sanitaria puede constituir un desafio importante para otras capas de la sociedad. Tener en cuenta específicamente a estos niños en campañas de vacunaciones y en otras acciones de sanidad pública, es prevención.

Además, existen otras poblaciones que han sido identificadas, mediante estudios epidemiológicos y de casos clínicos, como de riesgo más elevado para otros comportamientos disfuncionales específicos. Por ejemplo, aunque es conveniente abstenerse de relaciones sexuales prematrimoniales, la mayoría de los adolescentes no lo hacen. Hacia los 19 años de edad, más del 50 por ciento de mujeres y más de 64 por 
ciento de hombres han mantenido relaciones sexuales. Además, los adolescentes se caracterizan por no utilizar anticonceptivos. Menos de la mitad de los adolescentes sexualmente activos utilizan anticonceptivos con regularidad. Aún menos utilizan anticonceptivos que disminuyan la probabilidad de contraer enfermedades de transmisión sexual. El hecho de no practicar una actividad sexual segura expone al adolescente a embarazos no deseados y a una plétora de enfermedades, algunas de ellas mortales. Las intervenciones con este grupo de riesgo son claramente convenientes (Adams, Gullotta y Markstrom-Adams, 1994).

Por último, los profesionales de la prevención reconocen que las intervenciones únicas tienen muy pocas probabilidades de alcanzar el éxito. Los modelos de comportamiento se desarrollan durante largos períodos de tiempo. Suponer que una intervención única o una serie de intervenciones que solo duren varios meses dará como resultado un cambio de comportamiento para toda la vida, es poco realista. Ningún otro campo mantiene estas expectativas.

Por ejemplo, un individuo que ha recibido tratamiento contra el cáncer se considera curado si no vuelve a recaer durante un período de cinco años. Si recae al cabo de seis años se considera que es un caso nuevo. En ciertos programas de tratamiento como los de protección familiar intensiva, si transcurre un año después de una intervención sin que se vuelva a remitir a la familia al servicio de protección, se considera señal de que la intervención ha tenido éxito. Esta intervención de tratamiento no se considera fallida si la familia tratada vuelve a requerir tratamiento al cabo de 13 o más meses. Por último, consideremos la vacuna contra la gripe. No existe la expectativa de que una vacuna sirva para toda la vida contra todo tipo de formas de gripe o ni siquiera para una sola forma. La expectativa es que, para recibir alguna protección, la vacuna contra la gripe debe recibirse cada año.

En base a las normas acabadas de mencionar, programas como Head Start y muchísimos otros tienen un éxito impresionante. A la prevención se le deben pedir responsabilidades. Cabe esperar que demuestre su eficacia. Pero no debería juzgarse en base a unos patrones más severos que los existentes para otros campos.

\section{¿COMO SE PRODUCE LA PREVENCION PRIMARIA?}

\section{Actuando como consultores y colaboradores, los profesionales de la sanidad pública utilizan la tecnología de la educación, el fomento de la competencia, la organización comunitarial intervención sistémica y los cuidados naturales de la prevención primaria para fomentar el crecimiento de comportamientos funcionales en la sociedad}

Aunque las disciplinas profesionales tienen que desempeñar un papel de importancia vital en el fomento de la salud de la sociedad, siempre se debe tener presente que la responsabilidad de la salud recae en el individuo. El papel de los profesionales de la prevención estriba en generar oportunidades para la salud, en eliminar barreras que obstaculicen la salud y en alterar las circunstancias que la desalienten. No obstante, es el individuo quien finalmente decidirá entre ignorar o no estas oportunidades y escoger un estilo de vida disfuncional (malsano).

Los profesionales de la prevención desempeñan un papel auxiliar en esta alianza orientada hacia una buena salud, utilizando sus habilidades como asesores y colaboradores para mejorar la salud de una comunidad. El asesoramiento comporta el ofrecimiento de asistencia técnica por parte de profesionales de la sanidad pública que comparten sus habilidades, sus conocimientos y su formación con otras personas para prevenir comportamientos disfuncionales y fomentar comportamientos funcionales. Esto contrasta con la actividad práctica más activa que se realiza en la colaboración. La colaboración es una asociación activa de capacitadores profesionales que trabajan directamente con grupos, estimulando su participación y responsabilidad en temas que influyen en su salud. Las herramientas que utilizan los profesionales de 


\section{0}

la prevención para fomentar el crecimiento de comportamientos funcionales en la sociedad son la educación, el fomento de la competencia, la organización comunitaria/ intervención sistémica y los cuidados naturales (Gullotta, 1987).

La educación se basa en la creencia de que los seres humanos son criaturas racionales capaces de aprender a partir de sus propias experiencias y de las experiencias ajenas. En cada una de sus tres formas, puede utilizarse para facilitar el paso de un suceso de la vida a otro, o puede darse a los individuos para mejorar su bienestar. La educación puede adoptar la forma de información pública como la que se encuentra en anuncios de servicio público, películas, currículos y en este manuscrito. Puede adoptar la forma de guía de previsión que prepare a los individuos para la jubilación, el matrimonio, el parto o los muchos otros sucesos que marcan èl flujo de la vida. La educación también puede adoptar la forma de métodos conductuales como el biofeedback o la relajación progresiva, donde los individuos aprenden a controlar más sus cuerpos.

La educación es la herramienta utilizada con más frecuencia por la prevención aunque también es su instrumento más débil. La información y el cambio de actitudes que puede crear no conducen necesariamente a cambios de comportamiento. Ciertamente, en mi larga asociación con The Journal of Primary Prevention, no puedo recordar un solo caso de revisión de un manuscrito en el que una sola intervención educativa tuviera el efecto deseado de modificar un comportamiento disfuncional. Las intervenciones educativas aumentan el conocimiento, influyen en las actitudes, pero no modifican comportamientos. Es cuando la educación se combina con los siguientes tres instrumentos cuando el cambio tiene más probabilidades de ocurrir. Ciertamente, sugeriría a mis colegas que una iniciativa sólo debe considerarse un método de prevención cuando contiene un reconocimiento de estos cuatro instrumentos y los pone en práctica.

El segundo instrumento de la prevención, el fomento de la competencia, se define como las actividades que fomentan la pertenencia a un grupo, ser apreciado como miembro de ese grupo y ser capaz de bacer una contribución significativa a la existencia y la continuidad de ese grupo. La literatura psicológica rebosa de ejemplos en los que individuos con un gran amor propio, un centro interno de control y un interés por la comunidad, están más relacionados con comportamientos sociales funcionales que individuos centrados en sí mismos. Las actividades que promueven la competencia social fomentan la resistencia, es decir, la capacidad de un individuo para resistir las adversidades sociales, emocionales y de salud, y de triunfar donde otros suelen fracasar. Este instrumento puede adoptar formas pasivas (intelectuales) como la formación asertiva, la educación afectiva o la instrucción religiosa. También puede adoptar formas más activas (físicas) como las que se encuentran en «Outward Bound»o «Rope» (Blumenkrantz y Gavazzi, 1993).

El fomento de la competencia comparte características similares a las de muchos de los instrumentos que se examinan en este manuscrito. La distinción entre la educación y el fomento de la competencia se refleja en los resultados. En el primer caso, el resultado es un aumento del conocimiento que conduce a cambios de actitud y de comportamiento. En el segundo caso, el resultado es una vinculación que conduce a una inversión en la sociedad.

El siguiente instrumento de la prevención es la organización comunitaria/intervención sistémica (OC/IS). En ocasiones, la capacidad de vivir la vida con eficacia se ve obstaculizada por fuerzas que se encuentran más allá de las capacidades personales. Estas fuerzas obstaculizan o limitan el acceso a oportunidades y opciones vitales. Este instrumento se uriliza para corregir estas injusticias. Los obstáculos se pueden eliminar mediante cualquiera de las tres maneras siguientes. $\mathrm{La}$ primera es la modificación o eliminación de barreras institucionalizadas. Por ejemplo, la prácrica relativamente reciente de permitir a los padres que estén presentes en la sala de partos es un ejemplo de eliminación de una barrera institucional que facilita el apego y los vínculos entre padre e hijo ya en el nacimiento. Un segundo ejemplo 
sería la práctica de ofrecer a los padres alojamiento nocturno para que acompañen a sus hijos hospitalizados. La segunda forma que puede adoptar la OC/IS es el desarrollo de recursos comunitarios. Un ejemplo de este desarrollo de recursos sería la modificación de edificios para permitir el acceso a todas las personas. El desarrollo de asociaciones de vecinos para mejorar las condiciones de vida de la comunidad es otro ejemplo más. El tercer método OC/IS es la acción judicial o legislativa que conduce a conseguir un grado de autoridad. Los esfuerzos de la National Association for the Advancement of Colored People en el campo de los derechos civiles, de Mothers Against Drunk Driving para una aplicación más estricta de las leyes contra la conducción bajo los efectos del alcohol y de grupos pro Disabled American Rights en apoyo de la legislación ADA, ilustran la eficacia que puede tener este instrumento en la modificación del comportamiento.

La organización comunitaria/intervención sistémica es el más poderoso de todos los instrumentos de prevención. Y, sin embargo, es el instrumento menos discutido por los profesionales de la salud mental. En contraste con la educación, que utiliza el conocimiento para efectuar cambios primero de actitud y después de comportamiento, la OC/IS utiliza la acción judicial, comunitaria y legislativa para efectuar cambios, prestando poca atención o cuidado a las actitudes. La acción comunitaria, sea en forma de modificar prácticas institucionales (instando a interrumpir el seguimiento de la capacidad de los escolares, por ejemplo), de fomentar cambios en el vecindario (velando por el cumplimiento de las normas de edificación) o de fomentar la acción legislativa (en forma de controles más estrictos sobre la posesión de armas) es un territorio que los científicos sociales han evitado en años recientes. Los programas efectivos de prevención primaria permiten a los individuos afectados enfrentarse a estos temas y trabajar para modificar estas condiciones sociales disfuncionales.

El cuarto instrumento de la prevención son los cuidados naturales. Cuidar de uno mismo, de otros, cuidarse mutuamente: ésta es la esencia de los cuidados naturales. La inmensa mayoría de los ciudadanos se va abriendo camino durante toda la vida sin buscar ningún consejo, guía o apoyo remunerado. Al contrario, esta asistencia se encuentra en el entorno sustentador que rodea al individuo y se obtiene de ese entorno. Este manantial de fuerza emocional puede proceder de varias fuentes. Cuando el apoyo social toma la forma de ayuda de enseñantes o del clero, se denomina cuidado nativo especializado. Esta expresión es un reconocimiento de que la sociedad ha mantenido expectativas de ayuda de una naturaleza emocional y física en relación a algunas profesiones que, históricamente, no han mantenido ninguna conexión educativa formal con las disciplinas de la salud mental. Aunque los enseñantes, el clero y la policía continúan desempeñando papeles que normalmente no están asociados a la salud mental, cada vez se ha prestado más atención al enriquecimiento de las habilidades de estas profesiones y a capacitarlas para fomentar la salud pública. Los grupos de autoayuda mutua son otra forma de cuidado. Se produce cuando un individuo es a la vez prestador y receptor de cuidados en un grupo que no está conducido por un profesional de la salud mental. Así como todas las formas anteriores de cuidados comportaban conductas como compartir conocimientos, compartir experiencias, ofrecer una comprensión compasiva, compañerismo, $y$, cuando es necesario, enfrentamiento (Bloom, en preparación; Cowen, 1982), esta forma final de cuidado es de naturaleza individual. El cuidador nativo se hace responsable de su vida y, como he solicitado repetidamente a mis estudiantes a través de los años, invierte en la vida (salud) de por lo menos otro individuo.

\section{CONCLUSION}

Es de esperar que esta breve revisión de la prevención primaria conduzca al lector a otros trabajos sobre el tema. De los muchos libros excelentes dedicados a este 
campo, me inclino por remitir al lector a los siguientes compendios: Principles of Preventive Psychiatry (Caplan, 1964), Primary Prevention of Psychopathology Volume 1: The Issues (Albee y Joffe, 1977), Primary Prevention: The Possible Science (Bloom, 1981), Concepts of Primary Prevention: A Framework for Program Development (Goldston, 1987), 14 Ounces of Prevention (Price, Cowen, Lorion y Ramos-McKay, 1988) y Primary Prevention Practices (Bloom, en preparación). Recomiendo dos colecciones de libros de Sage Publication, una patrocinada por la Vermont Conference on the Primary Prevention of Psychopathology titulada Primary Prevention of Psychopatbology, y la otra 'patrocinada por la Child and Family Agency titulada Issues in Children's and Families' Lives, por su cobertura exhaustiva de aspectos de la prevención aplicados a temas específicos como la violencia familiar (Hampton, Gullotta, Adam, Potter y Weissberg, 1993).

Tengo la esperanza de que esta revisión de los principios de la prevención acelerará la incorporación de disciplinas nuevas a este prometedor campo de fomento de la salud. También cabe esperar que sirva para recordar que la prevención no pertenece a ninguna disciplina concreta. Ninguna organización profesional y ningún departamento estatal o federal tiene una potestad exclusiva sobre ella.

Finalmente, desconfiemos de las "panaceas»: como he comunicado en este artículo, no existe ninguna iniciativa específica que, por sí sola, pueda prevenir un solo comportamiento disfuncional. La prevención es exhaustiva, se extiende en el tiempo, pertenece al pueblo y su tecnología es genérica.

\section{Referencias}

Adams, G. R.; Gullotta, T. P., and Markstrom-Adams, C. (1994). Adolescent life experiences. Pacific Grove, CA: Brooks/Cole.

ALBEE, G. W. (1983). Psychopathology, prevention, and the just society. Joumal of Primary prevention, $4(1), 5-40$.

ALBEE, G. W. (1985). The argument for primary prevention. Journal of Primary Prevention, 5(4), $213-$ 219.

ALBEE, G. W. (1987). The rationale and need for primary prevention. In S. E. Goldston (Ed.) Concepts of primary prevention: A framework for program development. Los Angeles, CA: California Department of Mental Health.

AlBEE, G. W., and GULLOTTA, T. P. (1986). Facts and fallacies about primary prevention. Journal of Primary Prevention, 6(4), 207-218.

ALbeE, G. W., and Joffe, J. M. (1977). Primary prevention of prychopathology Volume 1: The issues. Hanover, New Hampshire: University Press of New England.

BLOOM, M. (1981). Primary prevention: The possible science. Englewood Cliffs. N. J.: Prentice-Hall.

BLOOM, M. (in progress). Primary prevention practices. Author.

BlumENKRANTZ, D. G., and GAVAZZI, S. M. (1993). Guiding transitional events for children and adolescents through a modern day rite of passage. Journal of Primary Prevention, 13(3), 199-212.

CAPLAN, G. (1964). Principles of preventive psychiatry. N.Y.: Basic Books.

COMMITEE ON PREVENTIVE PSYCHIATRY (1980). Mental bealth and primary medical care. N.Y., N.Y.: Group for the Advancement of Psychiatry.

COWEN, E. L. (1892). Help is where you find it: Four informal helping groups. American Psychologist, 37, 385-395.

Danish, S. J.; Petipas, A. J., and Hale, B. D. (1990). Sport as a context for developing competence. In T. P. Gullotta, G. R. Adams, and R. Montemayor (Eds.), Developing social competence in adolescence. Newbury Park, CA: Sage.

GOL.DSTON, S. E. (Ed.) (1987). Concepts of primary prevention: A framework for program development. Los Angeles, CA: California Department of Mental Health.

GuLLOTT, T. P. (1987). Prevention's technology. Journal of Primary Prevention, 8(1\&2), 4-24.

Hampton, R. L.; Gullota, T. P.; Adams, G. R.; Potter, E. H., and Weissberg R. P. (1993). Issues in children's and families lives Volume 1: Family violence. Newbury Park, CA: Sage.

HOLDEN, C. (1987). Is alcoholism treatment effective? Science, 236, 20-22.

Holden, C. (1991). Depression: The new isn't depressing. Science, 254, 1.450-1.452.

JASON, L. A.; KURASAKI, K. S.; NeUSON, L., and GARCIA, C. (1993). Training parents in a preventive intervention for transfer children. Journal of Primary Prevention, 13(3), 213-227. 
Klein, D. C., and Goldston, S. E. (Eds.) (1997). Primary prevention: An idea whose time has come. Washington, DC: National Institute of Mental Health.

National Commission on ChILdRen, DC: U. S. Government Printing Office.

PEELE, S. (1986). The implications and limitations fo genetic models of alcoholism and other addictions. Journal of Studies on Alcobol, 47(1), 63-73.

PLOMIN, R. (1990). The role of inheritance in behavior. Science, 248, 183-188.

PRICE, R.; COWEN, E.; LORION, R., and RAMOS-MCKAY, J. (Eds.) (1988). 14 ounces of prevention: A casebookfor practitioners. Washington, DC: American Psychological Association.

\section{El qué, el quién, el por qué, el dónde, el cuándo y el cómo de la prevención primaria Thomas P. Gullotta $C L \& E, 1995,27, p p .105-113$}

Resumen: En este artículo se examinan los principios básicos y genéricos de la prevención primaria. Se describen sus elementos esenciales y se explica cómo pueden utilizarse la tecnología de prevención de la educación, la competencia social, la organización comunitaria/intervención sistémica y los cuidados naturales, para prevenir comportamientos disfuncionales $y$, al mismo tiempo, fomentar estilos de vida sanos.

Articulo original: The What, Who, Why, Where, When and How of Primary Prevention. The Journal of Primary Prevention, 15 (1), pp. 5-13 (1994). Reproducido con autorización de Human Sciences Pres, Inc. (Subsidiary of: Plenum Publishing Corp.). Traducción de Genis Sánchez Barberán.

Dirección: Thomas P. Gullotta. Child and Family Agency, 255 Hempstead Street, New London, CT. 06320.

(C) PERMISOS PARA CITAR O REPRODUCIR EN OTRAS FUENTES: Se pueden citar libremente hasta 500 palabras. Para reproducir una porción de texto mayor, figuras o ilustraciones, se deberá pedir permiso por escrito a la revista, especificando el uso al que se destina el texto. En todos los casos, se deberá citar el copyright de $C L \& E$. En el caso de artículos o textos que hayan sido a su vez reproducidos en $C L \& E$ los interesados deberán dirigirse tanto a los detentadores del copyright original como a $C L \& E$, en el caso de que se quiera hacer uso de la traducción. FOTOCOPIAS: Para todo lo relacionado con el uso mediante fotocopia del material de esta revista, deberán dirigirse a: CEDRO, C/ José Marañón, 10, 3. Izda. Tel. 594 15 75. Fax 4453567 